\title{
Dawning precision treatment for gastric cancer: The latest biomarkers
}

\author{
Keran Sun, Huifang Lv, BeiBei Chen, Caiyun Nie, Jing Zhao, Saiqi Wang, \\ Jianzheng Wang, Weifeng Xu, Xiaobing Chen \\ Department of Gastroenterology, Affiliated Tumor Hospital of Zhengzhou University, Zhengzhou 450000, Henan \\ Province, China
}

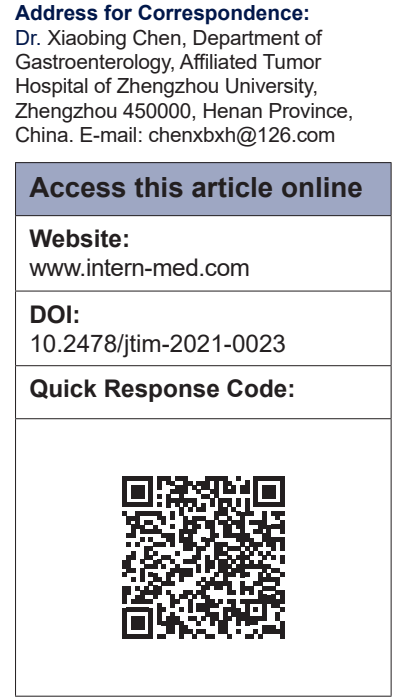

Recently, the International Agency for Research on Cancer (IARC) of the World Health Organization released the latest data about the global cancer burden in 2020 . The number of confirmed gastric cancer (GC) cases in the world is 1.09 million, accounting for $5.6 \%$ and ranking the fifth among all cancers. Immunotherapy has ushered in an era of precision treatment. ${ }^{[1]}$ Keynote-059 ${ }^{[2]}$, Keynote-061 ${ }^{[3]}$, Keynote-062, attraction- $02^{[4]}$, and other studies have repeatedly verified the effectiveness of PD-1 therapy. In addition, ANTXR1, IDO1, TIM-3, and LAG-3 have been found as functional biomarkers similar to PD-1, and their expression was related to the overall survival. ${ }^{[5]}$ Therefore, these biomarkers are likely to brew new precision treatments for GC in the future.

In 2019, Sotoudeh et al ${ }^{[6]}$ have found that ANTXR1 is expressed in both malignant tumor cells and tumor endothelial cells, making it a potential treatment target. They evaluated the relationship between ANTXR1 expression and the Lauren's classification of primary Gastric Cancer (GC), patients' neoadjuvant chemotherapy, radiotherapy history, and patients' overall survival. The results showed that ANTXR1 was less expressed in intestinal adenocarcinoma cells and highly expressed in adjacent normal endothelial cells; meanwhile, this low expression was associated with a poor overall survival and this high expression with a history of neoadjuvant therapy. In contrast, Cai et al. ${ }^{[7]}$ have found that ANTXR1 is overexpressed in GC and its overexpression is related to poor prognosis. In addition, the high protein level of ANTXR1 expression is positively correlated with many clinicopathological parameters of GC patients. ANTXR1 promotes GC progression by activating PI3K/AKT/ mTOR signal pathway. ANTXR1 promotes the proliferation, cycling, invasion, and migration, and inhibits the apoptosis of GC cells. ANTXR1 may be a new prognostic biomarker and potential therapeutic target.

As tumor develops, neovascularization is induced in the surrounding non-malignant tissues. New blood vessels provide oxygen and nutrients for tumor cells to sustain their growth, and an escape pathway for tumor metastasis. Therefore, curbing tumor-induced pathological angiogenesis has become the main target of anticancer therapy. ${ }^{[8]}$ However, the targeted drugs can also block the formation of normal blood vessels. ANTXR1 is a highly conserved cell surface protein overexpressed in vessels invaded by tumors, including melanoma, breast cancer, colon cancer, and lung cancer. ${ }^{[9,10]}$ Studies have found that suppressing ANTXR1 can selectively inhibit tumor-induced pathological angiogenesis, which endows it with a wide range of antitumor activities without cytotoxicity. ${ }^{[1]}$

IDO1 inhibits host anti-tumor immune response by depleting tryptophan in the tumor microenvironment. At present, various drugs can block IDO enzyme to reduce tryptophan consumption and enhance $T$ cells to promote tumor-specific immune response. ${ }^{[12]}$ In 2019, using the transcriptional data of gastric cancer cell line Cancer Cell Line Encyclopedia (CCLE), Xiang et al. ${ }^{[13]}$ established a weighted gene coexpression network (WGCNA) to explore the mechanism of IDO1 in 
GC development in vivo and in vitro. WGCNA analysis showed that IDO1 was enriched in extracellular matrix, collagen metabolism, and catabolism. Among the five HUB genes (Ax1, SGCE, COL12A1, ANTXR1, and LOXL2), COL12A1 and LOXL2 were upregulated in GC tissues. The coefficient matrix diagram showed that IDO1 was positively correlated with six collagen genes. IDO1 gene knockout reduced $\mathrm{mRNA}$ and protein levels of LOXL2, COL6A1, COL6A2, and COL12A1 in GC cells. Among them, knockout of COL12A1 exhibited stronger inhibitory effect on cell migration than that of other genes. IDO1 and COL12A1 cooperated to promote tumor cell migration through positive feedback maintained by MAPK pathway. Studies have shown that IDO1 and COL12A1 synergistically promote the metastasis of GC, suggesting that both can be used as new therapeutic targets.

The expression level of TIM-3 is significantly higher in paracancerous and paracancerous gastric tissues than in the peripheral blood, and is higher in cancerous tissues than in paracancerous tissues. Besides, the expression of TIM-3 increases with GC TNM stage. TIM-3 signaling pathway in $T$ cells can activate to inhibit the secretion of interferon- $\gamma$ and tumor necrosis factor- $\alpha$. The results of nude mouse models showed that the activation of TIM-3 signal pathway reduces the inhibitory effect of $\mathrm{T}$ cells on tumor growth. In GC, the expression level of TIM-3 on the surface of tumor infiltrating $\mathrm{T}$ cells is significantly increased, and the enhancement of TIM-3 signal may inhibit the function of T cells. ${ }^{[14]}$ In another study, Koyama et al. ${ }^{[15]}$ have found that the failure of anti-PD-1 therapy is associated with upregulation of TIM3 expression. Therefore, TIM3 may be a potential checkpoint, and anti-TIM3 monoclonal antibodies may ensure immune tolerance by blocking TIM3 receptors.

Clinical trials have tested the efficacy of three antiLAG-3 monoclonal antibodies (BMS-986016, LAG525, and MK-4280) combined with anti-PD-1 therapy. The results suggest that LAG-3 may be a potential target for immunotherapy. ${ }^{[16,17]}$ The value of peripheral blood-soluble LAG-3 (SLAG-3) in GC has been demonstrated by Li et al. ${ }^{[18]}$ in 2018. In this study, peripheral blood samples were collected from patients with GC and healthy people, and the levels of serum sLAG-3, carcinoembryonic antigen, IL-12, and interferon- $\gamma$ were measured and compared. At the same time, in vivo experiments were carried out in mice. The results showed that the expression of SLAG-3 in the peripheral blood of GC patients was low, and its expression was positively correlated with the expression of IL-12 and interferon- $\gamma$. The diagnostic value of SLAG-3 was higher than that of CEA. In vivo experiments showed that SLAG-3 could inhibit tumor growth and promote the secretion of CD8+ T cells, IL-12, and interferon- $\gamma$. In addition, SLAG-3 can prolong the overall survival time and improve the survival rate of tumor mice. The results showed that SLAG-3 had a positive regulatory effect on CD8 + T cells, IL-12, and interferon- $\gamma$, thus making it an ideal prognostic indicator and therapeutic target for GC.

With the rapid development of the next generation of sequencing, more and more molecular targets are being discovered. However, challenges should be resolved before these potential biomarkers and therapeutic targets are put to clinical use. Drugs with better efficacy are bound to emerge, so how to target them to individuals is also an issue that needs to be discussed. The immunotherapy for GC has only entered its first stage. How to use these targets to design therapeutic drugs and combine these drugs with surgery, radiotherapy, and chemotherapy to bring out the desired survival benefits is the ultimate goal of our research.

\section{Conflict of Interest}

None declared.

\section{Source of Funding}

This work is supported by the National Natural Science Foundation of China (81472714); Central Plains Thousand talents Plan-Central Plains leading Talent Project (204200510023).

\section{REFERENCES}

1. Marrelli D, Polom K, Pascale V, Vindigni C, Piagnerelli R, De Franco L, et al. Strong Prognostic Value of Microsatellite Instability in Intestinal Type Non-cardia Gastric Cancer. Ann Surg Oncol 2016; 23: 943-50.

2. Fuchs CS, Doi T, Jang RW, Muro K, Satoh T, Machado M, et al. Safety and Efficacy of Pembrolizumab Monotherapy in Patients With Previously Treated Advanced Gastric and Gastroesophageal Junction Cancer: Phase 2 Clinical KEYNOTE-059 Trial. JAMA Oncol 2018; 4: e180013.

3. Shitara K, Ozguroglu M, Bang YJ, Di Bartolomeo M, Mandalà M, Ryu $\mathrm{MH}$, et al. Pembrolizumab versus paclitaxel for previously treated, advanced gastric or gastro-oesophageal junction cancer (KEYNOTE-061): a randomised, open-label, controlled, phase 3 trial. Lancet 2018; 392: 123-33.

4. Chen LT, Satoh T, Ryu MH, Chao Y, Kato K, Chung HC, et al. A phase 3 study of nivolumab in previously treated advanced gastric or gastroesophageal junction cancer (ATTRACTION-2): 2-year update data. Gastric Cancer 2020;23:510-9.

5. Liu JB, Jian T, Yue C, Chen D, Chen, W, Bao TT, et al. Chemo-resistant Gastric Cancer Associated Gene Expression Signature: Bioinformatics Analysis Based on Gene Expression Omnibus. Anticancer Res 2019; 39: 1689-98.

6. Sotoudeh M, Shakeri R, Dawsey SM, Sharififard B, Ahmadbeigi N, Naderi M. ANTXR1 (TEM8) overexpression in gastric adenocarcinoma makes the protein a potential target of immunotherapy. Cancer Immunol Immunother 2019; 68:1597-1603.

7. Cai C, Dang W, Liu S, Huang L, Li Y, Li G, et al. Anthrax toxin receptor 1/ tumor endothelial marker 8 promotes gastric cancer progression through activation of the PI3K/AKT/mTOR signaling pathway. Cancer Sci 2020; 111: 1132-45.

8. Kerbel RS. Tumor angiogenesis. N Engl J Med 2008; 358: 2039-49. 
9 St Croix B, Rago C, Velculescu V, Traverso G, Romans KE, Montgomery E, et al. Genes expressed in human tumor endothelium. Science 2000; 289: 1197-202.

10. Nanda A, Carson-Walter EB, Seaman S, Barber TD, Stampfl J, Singh S, et al. TEM8 interacts with the cleaved C5 domain of collagen alpha 3(VI). Cancer Res 2004; 64: 817-20.

11. Chaudhary A, Hilton MB, Seaman S, Haines DC, Stevenson S, Lemotte PK, et al. TEM8/ANTXR1 blockade inhibits pathological angiogenesis and potentiates tumoricidal responses against multiple cancer types. Cancer Cell 2012; 21: 212-26.

12. Muller AJ, Prendergast GC. Marrying immunotherapy with chemotherapy: why say IDO?. Cancer Res 2005; 65: 8065-8.

13. Xiang Z, Li J, Song S, Wang J, Cai W, Hu W, et al. A positive feedback between IDO1 metabolite and COL12A1 via MAPK pathway to promote gastric cancer metastasis. J Exp Clin Cancer Res 2019; 38: 314.
14. Yu J, Zhang H, Sun S, Sun S, Li L. The effects of Tim-3 activation on T-cells in gastric cancer progression. Oncol Lett 2019; 17: 1461-6.

15. Koyama S, Akbay EA, Li YY, Herter-Sprie GS, Buczkowski KA, Richards WG, et al. Adaptive resistance to therapeutic PD-1 blockade is associated with upregulation of alternative immune checkpoints. Nat Commun, 2016, 7: 10501 .

16. Andrews LP, Marciscano AE, Drake CG, Vignali DA. LAG3 (CD223) as a cancer immunotherapy target. Immunol Rev 2017; 276: 80-96.

17. Lee SJ, Byeon SJ, Lee J, Park SH, Park JO, Park YS, et al. LAG3 in Solid Tumors as a Potential Novel Immunotherapy Target. J Immunother 2019; 42: 279-83.

18. Li N, Jilisihan B, Wang W, Tang Y, Keyoumu S. Soluble LAG3 acts as a potential prognostic marker of gastric cancer and its positive correlation with CD8+T cell frequency and secretion of IL-12 and INF-gamma in peripheral blood. Cancer Biomark 2018; 23: 341-51.

How to cite this article: Sun K, Lv H, Chen BB, Nie C, Zhao J, Wang $\mathrm{S}$, Wang J, Xu W, Chen X. Dawning precision treatment for gastric cancer: The latest biomarkers. J Transl Intern Med 2021; 9: 228-30. 